\title{
Cadernos de COMUNICAÇÃO
}

ufsm.br/ccomunicacao
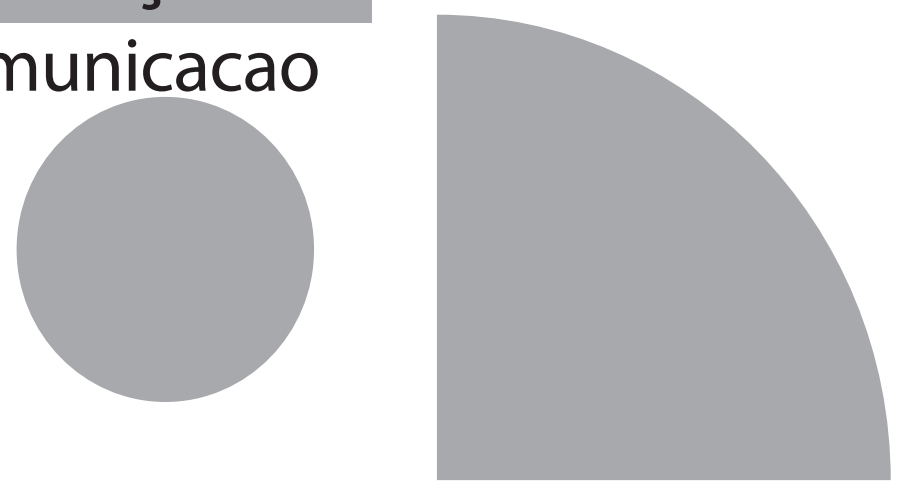



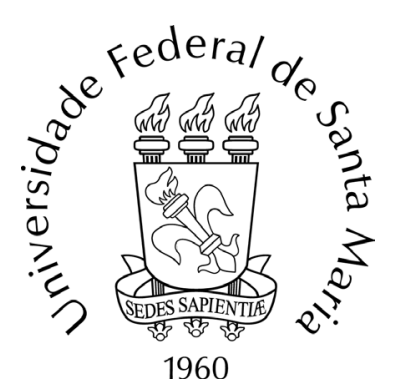

Centro de Ciências Sociais e Humanas Departamento de Ciências da Comunicação Programa de Pós-Graduação em Comunicação

\author{
Cadernos de \\ COMUNICAÇÃO \\ ISSN 1677-9061 \\ e-ISSN 2316-882X
}

Santa Maria - Rio Grande do Sul - Brasil

v. 20, n. 1, JAN - ABR 2016 
Cadernos de Comunicação / Universidade Federal de Santa Maria. Centro de Ciências Sociais e Humanas. Departamento de Ciências da Comunicação. Programa de Pós-Graduação em Comunicação. N. 1 (1996)Santa Maria, 1996-

Semestral (1996-2005/2011-2015) Quadrimestral (2016-)

ISSN 1677-9061

e-ISSN 2316-882X

Disponivel em: http://cascavel.ufsm.br/revistas/ojs-

2.2.2/index.php/ccomunicacao

1. Comunicação - Periódico. 2. Universidade Federal de Santa Maria - UFSM. 3. Centro de Ciências Sociais e Humanas - CCSH.

CDU $659.3(051)$

Ficha catalográfica elaborada por Fernando Leipnitz - CBR 10/1958

Biblioteca Central da UFSM

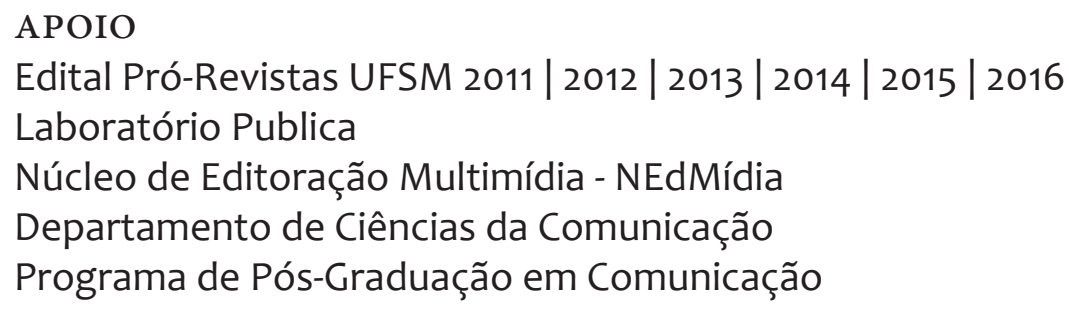




\section{CONSELHO EDITORIAL}

Ana Luiza Coiro de Moraes

Faculdade Cásper Líbero, Brasil

Bruno Souza Leal

Universidade Federal de Minas Gerais, Brasil

Eduardo Gutierrez

Pontificia Universidad Javeriana, Colômbia

Eneus Trindade

Universidade de São Paulo, Brasil

Fernanda Mauricio da Silva

Universidade Federal da Minas Gerais, Brasil

Gisela Cramer

Universidad Nacional de Colombia, Colômbia

Juçara Gorski Brittes

Universidade Federal de Ouro Preto, Brasil

Manuel Ramón González Herrera

Universidad Autónoma de Ciudad Juárez, México

Maria Angela Pavan

Universidade Federal do Rio Grande do Norte, Brasil

Maria Berenice da Costa Machado

Universidade Federal do Rio Grande do Sul, Brasil

Maria Lilia Dias de Castro

Universidade Federal de Santa Maria, Brasil

Mirta Varela

Universidad de Buenos Aires, Argentina

Monica Maronna

Universidad de la Republica, Uruguai

Nísia Martins do Rosário

Universidade Federal do Rio Grande do Sul, Brasil

Paulo César Castro

Universidade Federal do Rio de Janeiro, Brasil 


\section{EXPEDIENTE}

\section{UNIVERSIDADE FEDERAL DE SANTA MARIA}

REITOR: Prof. Paulo Afonso Burmann

VICE-REITOR: Prof. Paulo Bayard Dias Gonçalves

\section{CENTRO DE CIÊNCIAS SOCIAIS E HUMANAS}

DIRETOR: Dr. Mauri Leodir Löbler

VICE-DIRETOR: Wanderlei José Ghilardi

DEPARTAMENTO DE CIÊNCIAS DA COMUNICAÇÃO

Dra. Sandra Rubia Silva

Programa de Pós-GraduaçÃo em CoMunicaÇÃo

Dr. Cássio Santos Tomain

\section{CADERNOS DE COMUNICAÇÃO}

EDITOR CHEFE

Dr. Flavi Ferreira Lisbôa Filho

EDITORA GERENTE

Dra. Cristina Marques Gomes

CAPA/PROJETO GRÁFICO

Flavio Teixeira Quarazemin

EQUIPE EDITORIAL/ATUALIZAÇÃO DA CAPA/DIAGRAMAÇÃO

Bianca Silva Garcia 


\section{SUMÁRIO}

09 EDITORIAL

Flavi Ferreira Lisboa Filho

DOSSIÊ

13 TELEVISÃo PÚBLICA E DIREITOS HUMANOS:UMA ANÁLISE DA PROGRAMAÇÃO DA TV BRASIL E SEU PAPEL NA DIFUSÃO E NA PROMOÇÃO DO DEBATE AFIRMATIVO SOBRE A DECLARAÇÃO UNIVERSAL DOS DIREITOS HUMANOS

Waldelio Pinheiro do Nascimento Júnior

37 A TV CULTURA E SEU PAPEL NO PROCESSO DE IMPLANTAÇÃO DA MULTIPROGRAMAÇÃO NO BRASIL

Vivianne Lindsay Cardoso, Juliano Maurício Cardoso

57 A SEGUNDA TELA E AS CONVERSAÇÕES NAS REDES SOCIAIS ANÁLISE DO COMPORTAMENTO DAS TVS PÚBLICAS DE MINAS GERAIS Soraya Ferreira, Isabela Norton

81 INFORMAÇÃo E COMUNICAÇÃO REGIONAL NA PROGRAMAÇÃo DA TELEVISÃO EDUCATIVA DE BLUMENAU

Rafael Jose Bona

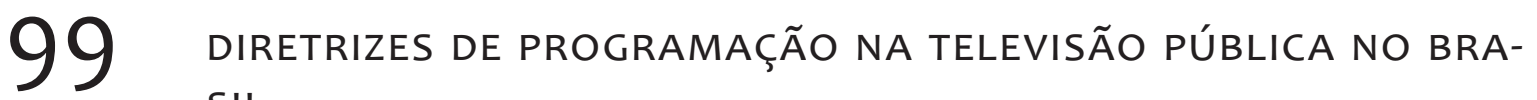
SIL

Tiago Nunes Severino

117 REDEIFES: UMA PERSPECTIVA CONVERGENTE E VIÁVEL DE UMA REDE INTERATIVA DE COMUNICAÇÃO HORIZONTAL E COLABORATIVA DAS IFES

Sérgio Nazaré de Sá Meyer Duque Estrada, Carlos Rocha, Francisco José Daher Junior

\section{ARTIGOS LIVRES}

137

O IMPERATIVO DA CULTURA DIGITAL:ENTRE NOVAS TECNOLOGIAS E ESTUDOS CULTURAIS

Sandro Faccin Bortolazzo 
153 ASOCIACIONES DE MIGRANTES ANDINOS EN LA WEB. RELEVANCOMUNITARIOS

Cecilia Melella

\section{COMUNICAÇÃO DE RESULTADOS DE PESQUISA}

175 DONA DE CASA E DA PRÓPRIA VIDA? LEITURAS SOBRE O TRABALHO FEMININO NA PUBLICIDADE POR MULHERES DA NOVA CLASSE TRABALHADORA

Milena Freire Oliveira-Cruz

185 O LIVRO E O AXÉ, O ERÊ COM GANGA ZUMBA: REPRESENTAÇÕES AFRO-BRASILEIRAS NA PRODUÇÃO EDITORIAL DIDÁTICA VOLTADA À LEI 10.639/2003 E APROPRIAÇÃO DOS PROFESSORES Fernanda de Araújo Patrocinio

195 CONFIGURAÇÃo dA AUTOPROMOCIONALIDAdE TELEVISUAL: ESTRATÉGIAS DE LANÇAMENTO DE UM PRODUTO MIDIÁTICO EM DIFERENTES PLATAFORMAS

Janderle Rabaiolli

205

A CIRCULAÇÃo dE SENTIDOS EM "EU NÃO MEREÇO SER ESTUPRADA": UMA LEITURA DO ACONTECIMENTO MIDIATIZADO Marlon Santa Maria Dias 
Caros Leitores,

Na edição v. 20, n.1 (jan-abr) de 2016, trazemos uma série de novidades. A partir de 2016 assumiu como editora-gerente a profa. Dra. Cristina Marques Gomes, seu doutorado em Ciência da Informação pela Escola de Comunicações e Artes da Universidade de São Paulo (USP) com a equivalência ao grau de Doutora em Tecnologia e Sistemas de Informação pela Escola de Engenharia da Universidade do Minho (Portugal), assim como seu doutorado Sanduíche - Programa Erasmus Mundus External Cooperation Window - Projecto ISAC - Improving Skills Across Continents, contribuem significativamente para qualificação da Revista Cadernos de Comunicação. Além disso, conseguimos atribuir o DOI para todos os artigos publicados das edições do ano de 2011 para cá. Estamos indexados no DOAJ, Latindex, Sumarios.org, Portcom e Diadorim. Tendo em conta os critérios de avaliação do qualis, também consideramos que $50 \%$ dos nossos autores ou co-autores, por edição, sejam doutores e para evitar a endogenia procuramos publicar artigos de autores de outras instituições nacionais e do estrangeiro. Um das principais mudanças foi a alteração na periodicidade de semestral para quadrimestral.

Reavaliamos nossa política de seções e passamos a operar em quatro modalidades, sejam elas: "Artigos", já tradicional e anteriormente chamada "temas livres"; "Dossiê", conforme propostas e demandas pertinentes ao campo da comunicação; "Comunicação de resultados de pesquisa", que procura dar visibilidade à produção científica desenvolvida junto ao Programa de Pós-Graduação em Comunicação da Universidade Federal de Santa Maria; e "Relatos de caso", em que alunos de iniciação científica ou recém-graduados poderão publicar em parceria com seus orientadores.

Para esta edição as professoras doutoras Ana Luiza Coiro Moraes da Faculdade Cásper Líbero/SP e Nádia Maria Weber dos Santos, Pesquisadora do Instituto Histórico e Geográfico do Rio Grande do Sul, organizaram um dossiê temático sobre "Televisão pública, redes de educação e cultura" com artigos cujas reflexões se dirigem aos estudos sobre TVs públicas, suas grades de programação, seus recursos imagéticos e visuais, seus regimes de identidade e representação dos poderes públicos a que se vinculam. Além disso, o dossiê traz pesquisas que debatam o pa- 
pel das Tvs públicas na promoção da cultura e na formação e conexão com variadas redes de educação, dentre outros temas que remetam, sobretudo, ao seu estatuto de dispositivo tecnológico que integra, conecta, configura e reconfigura o espaço público e que dá conta da memória das sociedades em que estão inseridos os arquivos audiovisuais pesquisados.

Esta edição apresenta 12 artigos, sendo seis deles no dossiê temático, dois na seção artigos e quatro referentes à comunicação de resultados de pesquisas. Informamos aos interessados que nosso gerenciamento está totalmente on-line e recebemos trabalhos em regime de fluxo contínuo.

Uma excelente leitura!

Flavi Ferreira Lisboa Filho 